\title{
Delay characteristics in discrete-time GI-G-1 queues with non-preemptive priority queueing discipline
}

\author{
Joris Walraevens, Bart Steyaert, Herwig Bruneel \\ SMACS Research Group, University of Ghent, Vakgroep TELIN (TW07V), \\ Sint-Pietersnieuwstraat 41, B-9000 Gent, Belgium. E-mail: jw@telin.rug.ac.be
}

\begin{abstract}
Priority scheduling for packets is becoming a hot topic, as attemps are being made to integrate voice services in existing data networks. In this paper, we consider a discrete-time queueing system with head-of-line (HOL) non-preemptive priority scheduling. Two classes of traffic will be considered, i.e., high-priority and lowpriority traffic, which both generate variable-length packets. We will derive expressions for the Probability Generating Function of the packet delay of the high-priority traffic and the low-priority traffic. From these, some performance measures (such as the mean value) will be derived. These will be used to illustrate the significance of priority scheduling and will be applied to an output queueing switch.
\end{abstract}

Key words: discrete-time queueing models, non-preemptive priority scheduling, packet delay, general service times

\section{Introduction}

In recent years, there has been much interest devoted to incorporating multimedia applications in packet-based networks. Different types of traffic need different QoS standards. For real-time applications, it is important that mean delay and delay-jitter are bounded, while for non real-time applications, the Loss Ratio (LR) is the restrictive quantity.

In general, one can distinguish two priority strategies, which will be referred to as Delay Priority and Loss Priority. Delay priority schemes attempt to guaran-

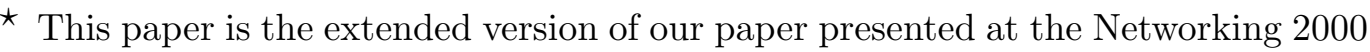
conference, Paris (May 14-19,2000).
} 
tee acceptable delay boundaries to delay-sensitive traffic (such as voice/video). This is achieved by giving it HOL priority over delay-insensitive traffic, and/or by sharing access to the server among the various traffic classes in such a way so that each can meet its own specific delay requirements. Several types of Delay priority (or scheduling) schemes (such as Weighted-Round-Robin (WRR), Weighted-Fair-Queueing(WFQ)) have been proposed and analyzed, each with their own specific algorithmic and computational complexity (see e.g. [5] and the references therein). On the other hand, Loss Priority schemes attempt to minimize the packet loss of loss-sensitive traffic (such as data). Again, various types of Loss Priority (or discarding) strategies (such as Push-Out Buffer (POB), Partial Buffer Sharing (PBS)) have been presented in the literature (see e.g. [15]). An overview of both types of priority schemes can be found in $[1]$.

In this paper, we will focus on a specific Delay priority scheme, namely, the non-preemptive HOL priority scheduling discipline. We assume that delaysensitive traffic has non-preemptive priority over delay-insensitive traffic, i.e., when the server becomes idle, a packet of delay-sensitive traffic, when available, will always be scheduled next, but newly arriving delay-sensitive traffic can not interrupt transmission of a delay-insensitive packet that has already commenced. This is a situation that occurs in some of the service models that have been proposed for packet-based networks, such as the Differentiated Service model for IP networks [16], where the Premium Service traffic class has (non-preemptive) HOL priority over all remaining traffic.

In the literature, there have been a number of contributions with respect to HOL priority scheduling. An overview of some basic HOL priority queueing models in continuous time can be found in Kleinrock [6], Miller [7] and Takagi [11] and the references therein. Khamisy et al. [3], Laevens et al. [4], Takine et al. [13] and Walraevens et al. [17] have studied discrete-time HOL priority queues with deterministic service times equal to one slot. Khamisy [3] analyzes the system contents for the different classes, for a queue fed by a two-state Markov modulated arrival process. Laevens [4] analyzes the system contents and cell delay in the case of a multiserver queue. In Takine [13], the system contents and the delay for Markov modulated high-priority arrivals and geometrically distributed low-priority arrivals are presented. Walraevens [17] studies the system contents and packet delay, in the special case of an output queueing switch with Bernoulli arrivals. Furthermore, non-preemptive HOL priority queues have been considered by Rubin et al. [8], Stanford [9], Sugahara et al. [10] and Takine et al. [12,14]. Rubin [8] studies the mean waiting time, for a discrete-time queue fed by an i.i.d. arrival process. Stanford [9] analyzes the interdeparture time distribution in a queue fed by a Poisson process in continuous time. In Sugahara [10], a non-preemptive queue in continuous time is presented, with a Switched Poisson Process arrival process for the high-priority packets. Finally, Takine $[12,14]$ studies a discrete-time 
MAP/G/1 queue, using matrix-analytic techniques.

In this paper, we analyze the packet delay of high- and low-priority traffic in a discrete-time single-server buffer with infinite buffer space for a nonpreemptive HOL priority scheme and per-slot i.i.d. arrivals. The transmission times of the packets generated by both types are assumed to be generally distributed. We will demonstrate that an analysis based on generating functions is extremely suitable for modelling this type of buffers with priority scheduling. From these generating functions, we can then easily calculate expressions for some interesting performance measures, such as the mean value and variance of the packet delay of both traffic types. These closed-form expressions require virtually no computational effort at all, and are well-suited for evaluating the impact of the various system parameters on the overall performance. This makes it possible to study the effect of priority scheduling and the impact of the non-preemptive priority scheduling on the high-priority traffic. These results could prove particularly interesting in packet-based networks, since packets in such networks have usually variable-length sizes and their service times have thus to be modelled as generally distributed variables. Furthermore, in many delay-sensitive real-time apllications (e.g. transmission of voice over packet-based networks) loss of information is caused mainly due to packets arriving too late at the receiver, rather than buffer overflow since the buffers used in packet-based networks (e.g. IP networks) are usually quite large. Therefore, the modelling of the buffer space as infinite is acceptable.

The remainder of this paper is structured as follows. In the following section, we present the mathematical model. In order to analyze the packet delay, it will be necessary to study the system contents at service initiation epochs, as done in section 3 . In section 4 we will then analyze the steady-state packet delay. In section 5 , we calculate the moments of the packet delay, while we discuss some of the results - obtained in the previous sections - in section 6. We apply our results to the specific case of an output queueing switch in section 7 and finally, some conclusions are formulated in section 8 .

\section{Mathematical model}

We consider a discrete-time single-server queueing system with infinite buffer space. Time is assumed to be slotted. There are 2 types of traffic arriving in the system, namely packets of class 1 and packets of class 2 . We denote the number of arrivals of class $j$ during slot $k$ by $a_{j, k}(j=1,2)$. Both types of packet arrivals are assumed to be i.i.d. from slot-to-slot and are characterized by the joint probability mass function

$$
a(m, n) \triangleq \operatorname{Prob}\left[a_{1, k}=m, a_{2, k}=n\right]
$$


and joint probability generating function (pgf) $A\left(z_{1}, z_{2}\right)$,

$$
A\left(z_{1}, z_{2}\right) \triangleq E\left[z_{1}^{a_{1, k}} z_{2}^{a_{2, k}}\right]
$$

Notice that the number of packet arrivals from different classes (within a slot) can be correlated. Further, we define the marginal pgf's of the number of arrivals from class 1 and class 2 during a slot by $A_{1}(z) \triangleq E\left[z^{a_{1, k}}\right]=A(z, 1)$ and $A_{2}(z) \triangleq E\left[z^{a_{2, k}}\right]=A(1, z)$ respectively. We furthermore denote the arrival rate of class $j(j=1,2)$ by $\lambda_{j}=A_{j}^{\prime}(1)$.

The service times of the class- $j$ packets are assumed to be i.i.d. and are characterized by the probability mass function

$$
s_{j}(m) \triangleq \operatorname{Prob}[\text { service of a class- } j \text { packet takes } m \text { slots], } m \geq 1 \text {, }
$$

and probability generating function $S_{j}(z)$,

$$
S_{j}(z)=\sum_{m=1}^{\infty} s_{j}(m) z^{m}
$$

with $j=1,2$. We furthermore denote the mean service time of a class- $j$ packet by $\mu_{j}=S_{j}^{\prime}(1)$. We define the load offered by class- $j$ packets as $\rho_{j} \triangleq \lambda_{j} \mu_{j}$ $(j=1,2)$. The total load is then given by $\rho_{T} \triangleq \rho_{1}+\rho_{2}$.

The system has one server that provides the transmission of packets. Class- 1 packets are assumed to have non-preemptive priority over class-2 packets, and within one class the service discipline is FCFS. Due to the priority scheduling mechanism, it is as if class- 1 packets are stored in front of class- 2 packets in the queue. So, if there are any class- 1 packets in the queue when the server becomes empty, the one with the longest waiting time will be served next. If, on the other hand, no class-1 packets are present in the queue at that moment, the class-2 packet with the longest waiting time, if any, will be served next. Since the priority scheduling is non-preemptive, service of a packet will not be interrupted by newly arriving packets.

\section{System contents at service initiation epochs}

To be able to analyze the packet delay, we will first analyze the system contents at the beginning of so-called start slots, i.e., slots at the beginning of which a packet (if available) can enter the server. Note that every slot during which the system is empty, is also a start slot. We denote the system contents of class- $j$ packets at the beginning of the $l$-th start slot by $n_{j, l}(j=1,2)$. Their joint pgf is denoted by $N_{l}\left(z_{1}, z_{2}\right)$, i.e.,

$$
N_{l}\left(z_{1}, z_{2}\right) \triangleq E\left[z_{1}^{n_{1, l}} z_{2}^{n_{2, l}}\right] .
$$


Clearly, the set $\left\{\left(n_{1, l}, n_{2, l}\right)\right\}$ forms a Markov chain, since the arrival process is i.i.d. and the buffer solely contains entire messages at the beginning of start slots. If $s_{l}^{*}$ indicates the service time of the packet that enters service at the beginning of start slot $l$ (which is - by definition - regular slot $k$ ) the following system equations can be established:

(1) If $n_{1, l}=n_{2, l}=0$ :

$$
\begin{aligned}
& n_{1, l+1}=a_{1, k} ; \\
& n_{2, l+1}=a_{2, k},
\end{aligned}
$$

i.e., the only packets present in the system at the beginning of start slot $l+1$ are the packets that arrived during the previous slot, i.e., start slot $l$.

(2) If $n_{1, l}=0$ and $n_{2, l}>0$ :

$$
\begin{aligned}
n_{1, l+1} & =\sum_{i=0}^{s_{l}^{*}-1} a_{1, k+i} ; \\
n_{2, l+1} & =n_{2, l}+\sum_{i=0}^{s_{l}^{*}-1} a_{2, k+i}-1,
\end{aligned}
$$

i.e., the class-2 packet in service leaves the system just before start slot $l+1 . s_{l}^{*}$ is characterized by probability mass function $s_{2}(m)$, since a class-2 packet enters the server at the beginning of start slot $l$.

(3) If $n_{1, l}>0$ :

$$
\begin{aligned}
& n_{1, l+1}=n_{1, l}+\sum_{i=0}^{s_{l}^{*}-1} a_{1, k+i}-1 ; \\
& n_{2, l+1}=n_{2, l}+\sum_{i=0}^{s_{l}^{*}-1} a_{2, k+i},
\end{aligned}
$$

i.e., the class- 1 packet in service leaves the system just before start slot $l+1 . s_{l}^{*}$ is characterized by probability mass function $s_{1}(m)$, since a class- 1 packet enters the server at the beginning of start slot $l$.

Using these system equations, we can derive a relation between $N_{l}\left(z_{1}, z_{2}\right)$ and $N_{l+1}\left(z_{1}, z_{2}\right)$. In the remainder, we define $\mathrm{E}[X\{Y\}]$ as $\mathrm{E}[X \mid Y] \operatorname{Prob}[Y]$. We proceed as follows, taking into account the statistical independence of the (set of) random variables $s_{l}^{*},\left(n_{1, l}, n_{2, l}\right)$ and $\left(a_{1, k+i}, a_{2, k+i}\right), i \geq 0$, respectively:

$$
\begin{aligned}
N_{l+1}\left(z_{1}, z_{2}\right) & \triangleq \mathrm{E}\left[z_{1}^{n_{1, l+1}} z_{2}^{n_{2, l+1}}\right] \\
& =\mathrm{E}\left[z_{1}^{a_{1, k}} z_{2}^{a_{2, k}}\left\{n_{1, l}=n_{2, l}=0\right\}\right]
\end{aligned}
$$




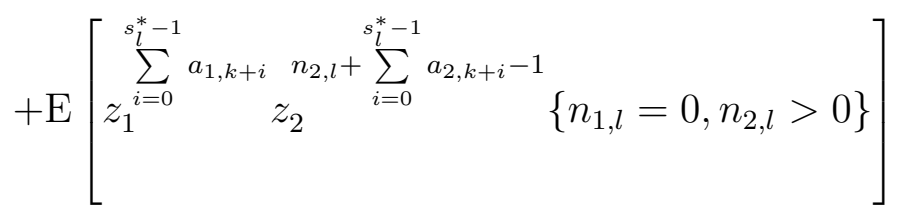

$$
\begin{aligned}
& +\mathrm{E}\left[\begin{array}{ccc}
n_{1, l}+\sum_{i=0}^{s_{l}^{*}-1} a_{1, k+i}-1 & n_{2, l}+\sum_{i=0}^{s_{l}^{*}-1} a_{2, k+i} & \\
z_{1} & z_{2} & \\
& &
\end{array}\right] \\
& =A\left(z_{1}, z_{2}\right) \operatorname{Prob}\left[n_{1, l}=n_{2, l}=0\right]+\frac{S_{2}\left(A\left(z_{1}, z_{2}\right)\right)}{z_{2}} \mathrm{E}\left[z_{2}^{n_{2, l}}\left\{n_{1, l}=0, n_{2, l}>0\right\}\right] \\
& +\frac{S_{1}\left(A\left(z_{1}, z_{2}\right)\right)}{z_{1}} \mathrm{E}\left[z_{1}^{n_{1, l}} z_{2}^{n_{2, l}}\left\{n_{1, l}>0\right\}\right] \\
& =A\left(z_{1}, z_{2}\right) N_{l}(0,0)+\frac{S_{2}\left(A\left(z_{1}, z_{2}\right)\right)}{z_{2}}\left[N_{l}\left(0, z_{2}\right)-N_{l}(0,0)\right] \\
& +\frac{S_{1}\left(A\left(z_{1}, z_{2}\right)\right)}{z_{1}}\left[N_{l}\left(z_{1}, z_{2}\right)-N_{l}\left(0, z_{2}\right)\right]
\end{aligned}
$$

We assume that the system is stable (implying that the equilibrium condition requires that $\left.\rho_{T}<1\right)$ and as a result $N_{l}\left(z_{1}, z_{2}\right)$ and $N_{l+1}\left(z_{1}, z_{2}\right)$ converge both to a common steady-state value:

$$
N\left(z_{1}, z_{2}\right) \triangleq \lim _{l \rightarrow \infty} N_{l}\left(z_{1}, z_{2}\right)
$$

By taking the $l \rightarrow \infty$ limit of equation (1), we obtain:

$$
\begin{aligned}
{\left[z_{1}-S_{1}\left(A\left(z_{1}, z_{2}\right)\right)\right] N\left(z_{1}, z_{2}\right)=} & z_{1} \frac{z_{2} A\left(z_{1}, z_{2}\right)-S_{2}\left(A\left(z_{1}, z_{2}\right)\right)}{z_{2}} N(0,0) \\
& +\frac{z_{1} S_{2}\left(A\left(z_{1}, z_{2}\right)\right)-z_{2} S_{1}\left(A\left(z_{1}, z_{2}\right)\right)}{z_{2}} N\left(0, z_{2}\right) .
\end{aligned}
$$

It now remains for us to determine the unknown function $N\left(0, z_{2}\right)$ and the unknown parameter $N(0,0)$. This can be done in two steps. First, we notice that $N\left(z_{1}, z_{2}\right)$ must be bounded for all values of $z_{1}$ and $z_{2}$ such that $\left|z_{1}\right| \leq 1$ and $\left|z_{2}\right| \leq 1$. In particular, this should be true for $z_{1}=Y\left(z_{2}\right)$, with $Y\left(z_{2}\right) \triangleq$ $S_{1}\left(A\left(Y\left(z_{2}\right), z_{2}\right)\right)$ and $\left|z_{2}\right| \leq 1$, since it follows from Rouché's theorem that there is exactly one solution $\left|Y\left(z_{2}\right)\right| \leq 1$ for all such $z_{2}$. Notice that $Y(1)$ equals 1. A stochastic interpretation of this function $Y(z)$ will be given in section 6 . The above implies that if we insert $z_{1}=Y\left(z_{2}\right)$ in equation (2), where $\left|z_{2}\right| \leq 1$, the left hand side of this equation vanishes. The same must then be true for the right hand side, yielding

$$
N\left(0, z_{2}\right)=N(0,0) \frac{z_{2} A\left(Y\left(z_{2}\right), z_{2}\right)-S_{2}\left(A\left(Y\left(z_{2}\right), z_{2}\right)\right)}{z_{2}-S_{2}\left(A\left(Y\left(z_{2}\right), z_{2}\right)\right)}
$$


The following expression for $N\left(z_{1}, z_{2}\right)$ can now be derived by combining equations (2) and (3):

$$
\begin{aligned}
N\left(z_{1}, z_{2}\right)= & N(0,0)\left[\frac{z_{1}\left(z_{2} A\left(z_{1}, z_{2}\right)-S_{2}\left(A\left(z_{1}, z_{2}\right)\right)\right)}{\left(z_{1}-S_{1}\left(A\left(z_{1}, z_{2}\right)\right)\right)\left(z_{2}-S_{2}\left(A\left(Y\left(z_{2}\right), z_{2}\right)\right)\right)}\right. \\
& +\frac{S_{2}\left(A\left(Y\left(z_{2}\right), z_{2}\right)\right)\left(S_{1}\left(A\left(z_{1}, z_{2}\right)\right)-z_{1} A\left(z_{1}, z_{2}\right)\right)}{\left(z_{1}-S_{1}\left(A\left(z_{1}, z_{2}\right)\right)\right)\left(z_{2}-S_{2}\left(A\left(Y\left(z_{2}\right), z_{2}\right)\right)\right)} \\
& \left.+\frac{A\left(Y\left(z_{2}\right), z_{2}\right)\left(z_{1} S_{2}\left(A\left(z_{1}, z_{2}\right)\right)-z_{2} S_{1}\left(A\left(z_{1}, z_{2}\right)\right)\right)}{\left(z_{1}-S_{1}\left(A\left(z_{1}, z_{2}\right)\right)\right)\left(z_{2}-S_{2}\left(A\left(Y\left(z_{2}\right), z_{2}\right)\right)\right)}\right] .
\end{aligned}
$$

Finally, in order to find an expression for $N(0,0)$, we put $z_{1}=z_{2}=1$ and use de l'Hôpital's rule in equation (4). Therefore, we need the first derivative of $Y(z)$ for $z=1$ and this is given by

$$
\begin{aligned}
Y^{\prime}(1) & =\mu_{1}\left(\lambda_{1} Y^{\prime}(1)+\lambda_{2}\right) \\
& =\frac{\lambda_{2} \mu_{1}}{1-\rho_{1}}
\end{aligned}
$$

We then obtain $N(0,0)$ :

$$
N(0,0)=\frac{1-\rho_{T}}{1-\rho_{T}+\lambda_{1}+\lambda_{2}} .
$$

Substituting the expression for $N(0,0)$ in $(4)$ gives a fully determined version of $N\left(z_{1}, z_{2}\right)$.

\section{Packet delay}

The packet delay is defined as the total time period a tagged packet spends in the system, i.e., the number of slots between the end of the packet's arrival slot and the end of its departure slot. We denote the steady-state delay of a tagged class- $j$ packet by $d_{j}$ and its pgf by $D_{j}(z)(j=1,2)$. Before deriving expressions for $D_{1}(z)$ and $D_{2}(z)$, we first define some notions and stochastic variables we will frequently use in this section. We denote the arrival slot of the tagged packet by slot $k$. If slot $k$ is a start slot, it is assumed to be start slot $l$. If slot $k$ is not a start slot on the other hand, the last start slot preceeding slot $k$ is assumed to be start slot $l$. We denote the number of class $j$ packets that arrive during slot $k$, but which are served before the tagged packet by $f_{j, k}(j=1,2)$. Since we only analyze the integer part of the delay, the precise time instant within the slot at which the tagged packet arrives, is not important. Only the order of service of all packets arriving in the same slot has to be specified. The class-1 packets will be serviced before the class- 2 packets, and within a class 


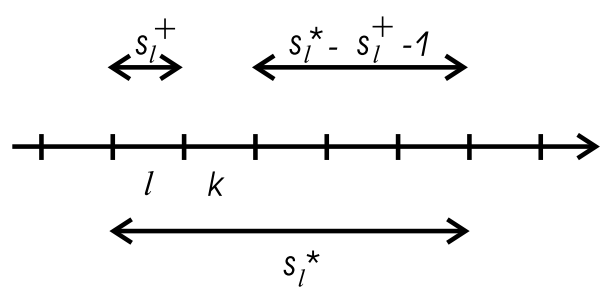

Fig. 1. Service time of the packet in service during slot $k$

the order of service is random. We furthermore denote the service time of the tagged class- $j$ packet by $\hat{s}_{j}(j=1,2)$. We finally denote the service time and the elapsed service time of the packet in service (if any) during the arrival slot of the tagged packet by $s_{l}^{*}$ and $s_{l}^{+}$respectively. The latter random variable is the amount of service that the packet being served has already received at the beginning of the tagged packet's arrival slot (see Figure 1).

\subsection{Delay of class-1 packets}

We tag a class-1 packet. There are 3 possibilities when the tagged packet arrives:

(1) The server is idle during slot $k$, yielding

$$
d_{1}=\sum_{m=1}^{f_{1, k}} s_{1, m}^{(k)}+\hat{s}_{1}
$$

with the $s_{1, m}^{(k)}$ 's the service times of the class-1 packets that arrived during slot $k$, but that are served before the tagged class-1 packet.

(2) A class-2 packet is in service during slot $k$ (implying that $n_{1, l}=0, n_{2, l}>$ $0)$, yielding

$$
d_{1}=\left(s_{l}^{*}-s_{l}^{+}-1\right)+\sum_{i=1}^{s_{l}^{+}} \sum_{m=1}^{a_{1, k-i}} s_{1, m}^{(k-i)}+\sum_{m=1}^{f_{1, k}} s_{1, m}^{(k)}+\hat{s}_{1},
$$

with the $s_{1, m}^{(k-i)}$ 's $\left(0 \leq i \leq s_{l}^{+}\right)$the service times of the class-1 packets that arrived during slot $k-i$. The residual service time of the packet in service during slot $k$ contributes in the first term, the service times of the class- 1 packets in the system at the beginning of slot $k$ contribute in the second term, the service times of the class-1 packets arrived during slot $k$, but served before the tagged class- 1 packet contribute in the third term, and finally the service time of the tagged class- 1 packet itself contributes in the last term. 
(3) A class-1 packet is in service during slot $k$ (i.e., $n_{1, l}>0$ ), yielding

$$
d_{1}=\left(s_{l}^{*}-s_{l}^{+}-1\right)+\sum_{m=1}^{n_{1, l}-1} \tilde{s}_{1, m}+\sum_{i=1}^{s_{l}^{+}} \sum_{m=1}^{a_{1, k-i}} s_{1, m}^{(k-i)}+\sum_{m=1}^{f_{1, k}} s_{1, m}^{(k)}+\hat{s}_{1} .
$$

The difference with the previous situation is that there may be multiple high-priority packets in the buffer (apart from the one in service) at the beginning of slot $l$, which will contribute to the tagged packet's delay. If we denote by $\tilde{s}_{1, m}$ the service times of the class- 1 packets already in the queue at the beginning of the ongoing service (thus without the packet in service during slot $k$ ), then this condition is quantified by the second term in the right-hand side of the above expression.

Using these equations, we can derive an expression for $D_{1}(z)$ :

$$
\begin{aligned}
& \left.D_{1}(z)=\mathrm{E}\left[z^{d_{1}}\{\text { no service }\}\right]+\mathrm{E}\left[z^{d_{1}} \text { \{service class-2 packet }\right\}\right] \\
& +\mathrm{E}\left[z^{d_{1}}\{\text { service class-1 packet }\}\right] \\
& =\left(1-\rho_{T}\right) \mathrm{E}\left[z^{\sum_{m=1}^{f_{1, k}} s_{1, m}^{(k)}+\hat{s}_{1}} \mid n_{1, l}=n_{2, l}=0\right] \\
& +\rho_{2} \mathrm{E}\left[z^{s_{l}^{*}-s_{l}^{+}-1+\sum_{i=1}^{s_{l}^{+}} \sum_{m=1}^{a_{1, k-i}} s_{1, m}^{(k-i)}+\sum_{m=1}^{f_{1, k}} s_{1, m}^{(k)}+\hat{s}_{1}} \mid n_{1, l}=0, n_{2, l}>0\right] \\
& +\rho_{1} \mathrm{E}\left[z^{s_{l}^{*}-s_{l}^{+}-1+\sum_{m=1}^{n_{1, l}-1} \tilde{s}_{1, m}+\sum_{i=1}^{s_{l}^{+}} \sum_{m=1}^{a_{1, k-i}} s_{1, m}^{(k-i)}+\sum_{m=1}^{f_{1, k}} s_{1, m}^{(k)}+\hat{s}_{1}} \mid n_{1, l}>0\right] \\
& =\mathrm{E}\left[z^{\sum_{m=1}^{f_{1, k}} s_{1, m}^{(k)}+\hat{s}_{1}}\right]\left\{1-\rho_{T}+\rho_{2} \mathrm{E}\left[z^{s_{l}^{*}-s_{l}^{+}-1+\sum_{i=1}^{s_{l}^{+}} \sum_{m=1}^{a_{1, k-i}} s_{1, m}^{(k-i)}} \mid n_{1, l}=0, n_{2, l}>0\right]\right. \\
& \left.+\rho_{1} \mathrm{E}\left[z^{s_{l}^{*}-s_{l}^{+}-1+\sum_{i=1}^{s_{l}^{+}} \sum_{m=1}^{a_{1, k-i}} s_{1, m}^{(k-i)}} \mid n_{1, l}>0\right] \mathrm{E}\left[z^{\sum_{m=1}^{n_{1, l}-1} \tilde{s}_{1, m}} \mid n_{1, l}>0\right]\right\} \\
& =\mathrm{E}\left[\left(S_{1}(z)\right)^{f_{1, k}+1}\right]\left\{1-\rho_{T}+\rho_{2} \frac{\mathrm{E}\left[\left(\frac{A_{1}\left(S_{1}(z)\right)}{z}\right)^{s_{l}^{+}} z^{s_{l}^{*}} \mid n_{1, l}=0, n_{2, l}>0\right]}{z}\right.
\end{aligned}
$$




$$
\begin{aligned}
& \left.+\rho_{1} \frac{\mathrm{E}\left[\left(\frac{A_{1}\left(S_{1}(z)\right)}{z}\right)^{s_{l}^{+}} z^{s_{l}^{*}} \mid n_{1, l}>0\right]}{z} \frac{N\left(S_{1}(z), 1\right)-N(0,1)}{(1-N(0,1)) S_{1}(z)}\right\} \\
& =F_{1}\left(S_{1}(z)\right) S_{1}(z)\left\{1-\rho_{T}+\rho_{2} \frac{S_{2}^{*}\left(\frac{\left.A_{1}\left(S_{1}(z)\right)\right)}{z}, z\right)}{z}\right. \\
& \left.+\rho_{1} \frac{S_{1}^{*}\left(\frac{\left.A_{1}\left(S_{1}(z)\right)\right)}{z}, z\right)}{z} \frac{N\left(S_{1}(z), 1\right)-N(0,1)}{(1-N(0,1)) S_{1}(z)}\right\}
\end{aligned}
$$

with $F_{1}(z) \triangleq E\left[z^{f_{1, k}}\right], S_{2}^{*}(x, z) \triangleq E\left[x^{s_{l}^{+}} z^{s_{l}^{*}} \mid n_{1, l}=0, n_{2, l}>0\right]$ and $S_{1}^{*}(x, z) \triangleq$ $E\left[x^{s_{l}^{+}} z^{s_{l}^{*}} \mid n_{1, l}>0\right]$. The random variable $f_{1, k}$ can be shown to have the following pgf (see e.g. [2]):

$$
F_{1}(z)=\frac{A_{1}(z)-1}{\lambda_{1}(z-1)}
$$

If a class- $j$ packet is in service during slot $k, s_{l}^{*}$ is characterized by the probability mass function $s_{j}(m)(j=1,2)$. Notice that the distributions of $s_{l}^{*}$ and $s_{l}^{+}$are correlated, since $s_{l}^{+}$is the elapsed part of the service time $s_{l}^{*}$ at the beginning of slot $k$. Considering these observations, one can derive the following expressions for $S_{j}^{*}(x, z)$ :

$$
S_{j}^{*}(x, z)=\frac{S_{j}(x z)-S_{j}(z)}{\mu_{j}(x-1)}
$$

with $j=1,2$. We now obtain the following expression for $D_{1}(z)$ from equation (7) together with equations (8) and (9):

$$
\begin{aligned}
D_{1}(z)= & \frac{S_{1}(z)\left(A_{1}\left(S_{1}(z)\right)-1\right)}{\lambda_{1}\left(S_{1}(z)-1\right)}\left\{1-\rho_{T}+\lambda_{2} \frac{S_{2}\left(A_{1}\left(S_{1}(z)\right)\right)-S_{2}(z)}{A_{1}\left(S_{1}(z)\right)-z}\right. \\
& \left.+\lambda_{1} \frac{S_{1}\left(A_{1}\left(S_{1}(z)\right)\right)-S_{1}(z)}{A_{1}\left(S_{1}(z)\right)-z} \frac{N\left(S_{1}(z), 1\right)-N(0,1)}{(1-N(0,1)) S_{1}(z)}\right\} .
\end{aligned}
$$

Using equation (4) in equation (10) gives us a fully determined version of $D_{1}(z)$ :

$$
D_{1}(z)=\frac{1-\rho_{1}}{\lambda_{1}} \frac{S_{1}(z)(z-1)}{z-A_{1}\left(S_{1}(z)\right)} \frac{A_{1}\left(S_{1}(z)\right)-1}{S_{1}(z)-1}\left(1-\frac{\rho_{2}}{1-\rho_{1}}+\frac{\rho_{2}}{1-\rho_{1}} \frac{S_{2}(z)-1}{\mu_{2}(z-1)}\right)
$$




\subsection{Delay of class-2 packets}

Because of the priority discipline, an expression for $d_{2}$ will be a bit more involved. We now tag a class-2 packet that enters the buffer during slot $k$. Let us refer to the packets in the system at the end of slot $k$, but that have to be served before the tagged packet as the "primary packets". So, basically, the tagged class- 2 packet can enter the server, when all primary packets and all class- 1 packets that arrived after slot $k$ (i.e., while the tagged packet is waiting in the queue) are transmitted. In order to analyze the delay of the tagged class2 packet, the number of class- 1 packets and class- 2 packets that are served between the arrival slot of the tagged class- 2 packet and its departure slot is important, not the precise order in which they are served. Therefore, in order to facilitate the analysis, we will consider an equivalent virtual system with an altered service discipline. We assume that from slot $k$ on, the order of service for class- 1 packets (those in the queue at the end of slot $k$ and newly arriving ones) is LCFS instead of FCFS in the equivalent system (the transmission of class-2 packets remains FCFS). So, a primary packet can enter the server, when the system becomes free (for the first time) of class-1 packets that arrived during and after the service time of the primary packet that predecessed it according to the new service discipline. Let $v_{1, m}^{(i)}$ denote the length of the time period during which the server is occupied by the $m$-th class- 1 packet that arrives during slot $i$ and its class-1 "successors", i.e., the time period starting at the beginning of the service of that packet and terminating when the system becomes free (for the first time) of class- 1 packets which arrived during and after its service time. Analogously, let $v_{2, m}^{(i)}$ denote the length of the time period during which the server is occupied by the $m$-th class- 2 packet that arrives during slot $i$ and its class-1 "successors". The $v_{j, m}^{(i)}$ 's $(j=1,2)$ are called sub-busy periods, caused by the $m$-th class- $j$ packet that arrived during slot $i$.

When the tagged class- 2 packet arrives, there are 3 possibilities:

(1) The server is idle during slot $k$, yielding

$$
d_{2}=\sum_{j=1}^{2} \sum_{m=1}^{f_{j, k}} v_{j, m}^{(k)}+\hat{s}_{2}
$$

i.e., $f_{1, k}$ class- 1 primary packets and $f_{2, k}$ class- 2 primary packets that arrived during slot $k$ and their class- 1 successors have to be served before the tagged class- 2 packet.

(2) A class-2 packet is in service during slot $k$, yielding

$$
d_{2}=\left(s_{l}^{*}-s_{l}^{+}-1\right)+\sum_{i=1}^{s_{l}^{*}-s_{l}^{+}-1} \sum_{m=1}^{a_{1, k+i}} v_{1, m}^{(k+i)}+\sum_{j=1}^{2} \sum_{m=1}^{f_{j, k}} v_{j, m}^{(k)}+\sum_{j=1}^{2} \sum_{i=1}^{s_{l}^{+}} \sum_{m=1}^{a_{j, k-i}} v_{j, m}^{(k-i)}
$$




$$
+\sum_{m=1}^{n_{2, l}-1} \tilde{v}_{2, m}+\hat{s}_{2},
$$

with the $\tilde{v}_{2, m}$ 's the sub-busy periods, caused by the $m$-th class- 2 packet already in the queue at the beginning of start slot $l$. The residual service time of the packet in service during slot $k$ contributes in the first term, the sub-busy periods of the class-1 packets arriving during the residual service time contribute in the second term, the sub-busy periods of the class- 1 and class- 2 packets arriving during slot $k$, but that have to be served before the tagged class-2 packet contribute in the third term, the sub-busy periods of the class- 1 and class- 2 packets that arrived during the elapsed service time contribute in the fourth term, the sub-busy periods of the class-2 packets already in the queue at the beginning of start slot $l$ contribute in the fifth term and finally the service time of the tagged class-2 packet itself contributes in the last term.

(3) A class-1 packet is in service during slot $k$, yielding

$$
\begin{aligned}
d_{2}= & \left.s_{l}^{*}-s_{l}^{+}-1\right)+\sum_{i=1}^{s_{l}^{*}-s_{l}^{+}-1} \sum_{m=1}^{a_{1, k+i}} v_{1, m}^{(k+i)}+\sum_{j=1}^{2} \sum_{m=1}^{f_{j, k}} v_{j, m}^{(k)}+\sum_{j=1}^{2} \sum_{i=1}^{s_{l}^{+}} \sum_{m=1}^{a_{j, k-i}} v_{j, m}^{(k-i)} \\
& +\sum_{m=1}^{n_{1, l}-1} \tilde{v}_{1, m}+\sum_{m=1}^{n_{2, l}} \tilde{v}_{2, m}+\hat{s}_{2},
\end{aligned}
$$

The difference with the previous situation is that there may be multiple high-priority packets in the buffer (apart from the one in service) at the beginning of slot $l$, which will contribute to the tagged packet's delay. If we denote by $\tilde{v}_{1, m}$ the sub-busy periods caused by the class- 1 packets already in the queue at the beginning of the ongoing service (thus without the packet in service during slot $k$ ), then this condition is quantified by the fifth term in the right-hand side of the above expression.

Using these equations, we can derive an expression for $D_{2}(z)$ :

$$
\begin{aligned}
& D_{2}(z)=\mathrm{E}\left[z^{d_{2}}\{\text { no service }\}\right]+\mathrm{E}\left[z^{d_{2}}\{\text { service class-2 packet }\}\right] \\
& +\mathrm{E}\left[z^{d_{2}}\{\text { service class-1 packet }\}\right] \\
& =\mathrm{E}\left[\sum^{j=1} \sum_{m=1}^{f_{j, k}} v_{j, m}^{(k)}+\hat{s}_{2}\right]\left\{1-\rho_{T}\right. \\
& +\rho_{2} \mathrm{E}\left[z^{s_{l}^{*}-s_{l}^{+}-1+} \sum_{i=1}^{s_{l}^{*}-s_{l}^{+}-1} \sum_{m=1}^{a_{1, k+i}} v_{1, m}^{(k+i)}+\sum_{j=1}^{2} \sum_{i=1}^{s_{l}^{+}} \sum_{m=1}^{a_{j, k-i}} v_{j, m}^{(k-i)} \mid n_{1, l}=0, n_{2, l}>0\right]
\end{aligned}
$$




$$
\begin{aligned}
& \mathrm{E}\left[z_{m=1}^{\sum_{2, l}^{-1} \tilde{v}_{2, m}} \mid n_{1, l}=0, n_{2, l}>0\right] \\
& +\rho_{1} \mathrm{E}\left[z^{\left.s_{l}^{*}-s_{l}^{+}-1+\sum_{i=1}^{s_{l}^{*}-s_{l}^{+}-1} \sum_{m=1}^{a_{1, k+i}} v_{1, m}^{(k+i)}+\sum_{j=1}^{2} \sum_{i=1}^{s_{l}^{+}} \sum_{m=1}^{a_{j, k-i}} v_{j, m}^{(k-i)} \mid n_{1, l}>0\right]}\right. \\
& \left.\mathrm{E}\left[z \sum_{m=1}^{n_{1, l}^{-1}} \tilde{v}_{1, m}+\sum_{m=1}^{n_{2, l}} \tilde{v}_{2, m} \mid n_{1, l}>0\right]\right\} .
\end{aligned}
$$

It is clear that the length of the sub-busy periods caused by class- 1 packets are i.i.d. and thus have the same pgf $V_{1}(z)$. Also the length of the sub-busy periods caused by class-2 packets are i.i.d., and their pgf is denoted by $V_{2}(z)$. Notice that $f_{1, k}$ and $f_{2, k}$ are correlated; in section 2 it was explained that $a_{1, k}$ and $a_{2, k}$ may be correlated as well. Equation (12) then becomes

$$
\left.\begin{array}{rl}
D_{2}(z)= & \mathrm{E}\left[V_{1}(z)^{f_{1, k}} V_{2}(z)^{f_{2, k}}\right] S_{2}(z)\left\{1-\rho_{T}\right. \\
& +\rho_{2} \frac{\mathrm{E}\left[\left(\frac{A\left(V_{1}(z), V_{2}(z)\right)}{z A_{1}\left(V_{1}(z)\right)}\right)^{s_{l}^{+}}\left(z A_{1}\left(V_{1}(z)\right)\right)^{s_{l}^{*}} \mid n_{1, l}=0, n_{2, l}>0\right]}{z A_{1}\left(V_{1}(z)\right)} \\
& \left.\frac{\mathrm{E}\left[V_{2}(z)^{\left.n_{2, l} \mid n_{1, l}=0, n_{2, l}>0\right]}\right.}{V_{2}(z)}\right\} \\
& +\rho_{1} \frac{\mathrm{E}\left[\left(\frac{A\left(V_{1}(z), V_{2}(z)\right)}{z A_{1}\left(V_{1}(z)\right)}\right)^{s_{l}^{+}}\left(z A_{1}\left(V_{1}(z)\right)\right)^{s_{l}^{*}} \mid n_{1, l}>0\right]}{z A_{1}\left(V_{1}(z)\right)} \\
& \left.\frac{\mathrm{E}\left[V_{1}(z)^{n_{1, l}} V_{2}(z)^{\left.n_{2, l} \mid n_{1, l}>0\right]}\right.}{V_{1}(z)}\right\} \\
= & F\left(V_{1}(z), V_{2}(z)\right) S_{2}(z)\left\{1-\rho_{T}\right. \\
& +\rho_{2} \frac{S_{2}^{*}\left(\frac{A\left(V_{1}(z), V_{2}(z)\right)}{z A_{1}\left(V_{1}(z)\right)}, z A_{1}\left(V_{1}(z)\right)\right)}{z A_{1}\left(V_{1}(z)\right)} \frac{N\left(0, V_{2}(z)\right)-N(0,0)}{(N(0,1)-N(0,0)) V_{2}(z)} \\
& +\rho_{1} \frac{N\left(V_{1}(z), V_{2}(z)\right)-N\left(0, V_{2}(z)\right)}{(1-N(0,1)) V_{1}(z)}
\end{array}\right\},
$$

with $F\left(z_{1}, z_{2}\right) \triangleq E\left[z_{1}^{f_{1, k}} z_{2}^{f_{2, k}}\right], S_{2}^{*}(x, z) \triangleq E\left[x^{s_{l}^{+}} z^{s_{l}^{*}} \mid n_{1, l}=0, n_{2, l}>0\right]$ and 
$S_{1}^{*}(x, z) \triangleq E\left[x^{s_{l}^{+}} z^{s_{l}^{*}} \mid n_{1, l}>0\right]$. The random variables $f_{1, k}$ and $f_{2, k}$ can be shown to have the following joint pgf (extension of a technique used in e.g. [2]):

$$
F\left(z_{1}, z_{2}\right)=\frac{A\left(z_{1}, z_{2}\right)-A_{1}\left(z_{1}\right)}{\lambda_{2}\left(z_{2}-1\right)}
$$

The $S_{j}^{*}(x, z)$ 's $(j=1,2)$ are again given by equation $(9)$. Finally, we have to find expressions for $V_{1}(z)$ and $V_{2}(z)$. These pgfs satisfy the following relations:

$$
V_{j}(z)=S_{j}\left(z A_{1}\left(V_{1}(z)\right)\right)
$$

with $(j=1,2)$. This can be understood as follows: when the $m$-th class $j$ packet that arrived during slot $i$ enters service, $v_{j, m}^{(i)}$ consists of two parts: the service time of that packet itself, and the service times of the class- 1 packets that arrive during its service time and of their class- 1 successors. This leads to equation (15).

Equation (13) together with equations (14) and (9) leads to:

$$
\begin{aligned}
D_{2}(z)= & \frac{A\left(V_{1}(z), V_{2}(z)\right)-A_{1}\left(V_{1}(z)\right)}{\lambda_{2}\left(V_{2}(z)-1\right)} S_{2}(z)\left\{1-\rho_{T}\right. \\
& +\lambda_{2} \frac{S_{2}\left(A\left(V_{1}(z), V_{2}(z)\right)-V_{2}(z)\right.}{V_{2}(z)\left(A\left(V_{1}(z), V_{2}(z)\right)-z A_{1}\left(V_{1}(z)\right)\right)} \frac{N\left(0, V_{2}(z)\right)-N(0,0)}{N(0,1)-N(0,0)} \\
& \left.+\lambda_{1} \frac{S_{1}\left(A\left(V_{1}(z), V_{2}(z)\right)-V_{1}(z)\right.}{V_{1}(z)\left(A\left(V_{1}(z), V_{2}(z)\right)-z A_{1}\left(V_{1}(z)\right)\right)} \frac{N\left(V_{1}(z), V_{2}(z)\right)-N\left(0, V_{2}(z)\right)}{1-N(0,1)}\right\},
\end{aligned}
$$

with $V_{j}(z)(j=1,2)$ implicitly given by equation (15). Using (4) in the former equation, we can finally obtain a fully determined version for $D_{2}(z)$ :

$$
D_{2}(z)=\frac{1-\rho_{T}}{\lambda_{2}} \frac{S_{2}(z)\left(A\left(V_{1}(z), V_{2}(z)\right)-A_{1}\left(V_{1}(z)\right)\right)}{z A_{1}\left(V_{1}(z)\right)-A\left(V_{1}(z), V_{2}(z)\right)} \frac{1-z A_{1}\left(V_{1}(z)\right)}{1-V_{2}(z)} .
$$

\section{Calculation of moments}

The functions $Y(z), V_{1}(z)$ and $V_{2}(z)$ can only be explicitly found in case of some simple arrival processes. Their derivatives for $z=1$, necessary to calculate the moments of the system contents and the cell delay, on the contrary, can be calculated in closed-form. For example, $Y^{\prime}(1)$ is given by equation (5) and the first derivatives of $V_{j}(z)$ for $z=1$ are given by

$$
V_{j}^{\prime}(1)=\frac{\mu_{j}}{1-\rho_{1}},
$$


with $j=1,2$. Let us define $C_{a_{j}} \triangleq \sqrt{\operatorname{Var}\left[a_{j, k}\right]} / \lambda_{j}$ (coefficient of variation of the number of per-slot class $j$ arrivals $), \rho_{a_{1} a_{2}} \triangleq \operatorname{Cov}\left[a_{1, k} a_{2, k}\right] / \sqrt{\operatorname{Var}\left[a_{1, k}\right] \operatorname{Var}\left[a_{2, k}\right]}$ (correlation between the number op per-slot arrivals of both classes) and $C_{s_{j}} \triangleq \sqrt{\operatorname{Var}[\text { service time class } j \text { packet] }} / \mu_{j}$ (coefficient of variation of the service times of class $j$ ), $j=1,2$. Now we can calculate the mean values of the packet delay of both classes by taking the first derivatives of the respective pgfs for $z=1$. We find

$$
E\left[d_{1}\right]=\frac{\mu_{1}}{2}+\frac{\rho_{1}\left(C_{a_{1}}^{2}+\mu_{1} C_{s_{1}}^{2}\right)}{2\left(1-\rho_{1}\right)}+\frac{\rho_{2}\left(\mu_{2} C_{s_{2}}^{2}+\left(\mu_{2}-1\right)\right)}{2\left(1-\rho_{1}\right)},
$$

for the mean packet delay of a class-1 packet and

$E\left[d_{2}\right]=\frac{\mu_{2}}{2}+\frac{\rho_{2} C_{a_{2}}^{2}}{2\left(1-\rho_{T}\right)}+\frac{\rho_{2} \mu_{2} C_{s_{2}}^{2}}{2\left(1-\rho_{T}\right)\left(1-\rho_{1}\right)}+\frac{\rho_{1}\left(\rho_{1} C_{a_{1}}^{2}+\mu_{1} C_{s_{1}}^{2}\right)}{2\left(1-\rho_{T}\right)\left(1-\rho_{1}\right)}-\frac{\rho_{1}\left(\mu_{2}-1\right)}{2\left(1-\rho_{1}\right)}+\frac{\rho_{1} C_{a_{1}} C_{a_{2}} \rho_{a_{1} a_{2}}}{1-\rho_{T}}$,

for the mean packet delay of a class-2 packet.

In a similar way, expressions for the variance (and higher order moments) can be calculated by taking the appropriate derivatives of the respective generating functions as well. Although these expressions are elaborate, implementing them in a computer tool is straightforward.

\section{Discussion of the results}

\subsection{The function $Y(z)$}

The function $Y(z)$ is the pgf of the stochastic variable $y$, which can be defined as the number of low priority packets that arrive during a sub-busy period caused by a high-priority packet in the alternative service discipline, described in subsection 4.2. If at the beginning of slot $k$ a high-priority packet with service time $\tilde{s}_{1}^{(k)}$ enters the server, a new sub-busy period starts. If we denote the number of class- 2 packets that arrive during this sub-busy period by $\tilde{y}^{(k)}$, then

$$
\tilde{y}^{(k)}=\sum_{i=0}^{\tilde{s}_{1}^{(k)}-1}\left(a_{2, k+i}+\sum_{m=1}^{a_{1, k+i}} y_{m}^{(k+i)}\right),
$$

with $y_{m}^{(k+i)}$ the number of class-2 packets that arrive during the sub-busy period started by the $m$-th class 1 packet that arrives during slot $k+i$. Naturally, all $y_{m}^{(k+i)}$ have the same distribution as $\tilde{y}^{(k)}$ (since the lengths of all sub-busy periods are also i.i.d.) and their pgf is thus indeed given by $Y(z)=S_{1}(A(Y(z), z))$, as immediately follows from (19) assuming that a stationary regime is reached. 


\begin{tabular}{||c|l|l||}
\hline class 1 parameters & $\mathrm{E}\left[d_{1}\right]$ & $\mathrm{E}\left[d_{2}\right]$ \\
\hline$\rho_{1}\left(\mu_{1}\right.$ constant $)$ & $\sim 1 /\left(1-\rho_{1}\right)$ & $\sim 1 /\left(1-\rho_{1}\right)+A_{1} /\left(1-\rho_{1}-\rho_{2}\right)$ \\
\hline$\rho_{1}\left(\lambda_{1}\right.$ constant $)$ & $\sim \rho_{1}+B_{1} /\left(1-\rho_{1}\right)$ & $\sim 1 /\left(1-\rho_{1}\right)+C_{1} /\left(1-\rho_{1}-\rho_{2}\right)$ \\
\hline$\mu_{1}\left(\rho_{1}\right.$ constant $)$ & $\sim \mu_{1}$ & $\sim \mu_{1}$ \\
\hline$C_{a_{1}}$ & $\sim C_{a_{1}}^{2}$ & $\sim C_{a_{1}}^{2}+D_{1} C_{a_{1}}$ \\
\hline$C_{s_{1}}$ & $\sim C_{s_{1}}^{2}$ & $\sim C_{s_{1}}^{2}$ \\
\hline & &
\end{tabular}

Table 1

Dependence of the mean delay of both priority classes on the system parameters of the high-priority class $\left(A_{1}, . ., D_{1}\right.$ are constants)

\subsection{The function $D_{1}(z)$}

Equation (11) shows that the delay of the class-1 packets is the sum of two statistically independent terms: the first term is the delay of a class- 1 packet in the absence of class-2 packets (e.g. [2]), while the second term accounts for the situation where a class-2 packet is being served when the tagged class- 1 packet enters the buffer, in which case a class- 2 residual service time must be added.

\subsection{The mean packet delay}

Obviously, the delay of a packet contains its own servicetime. So, the mean delay of class- $j$ packets is bigger than or equal to $\mu_{j}(j=1,2)$. This can also be seen from the expressions (17)-(18). $C_{a_{j}}^{2}$ can not be less than $\left(1-\lambda_{j}\right) / \lambda_{j}$ (this value is reached in the situation that the number of class- $j$ packet arrivals per slot is 0 (with probability $1-\lambda_{j}$ ) or 1 (with probability $\lambda_{j}$ ), $j=1,2$, which is clearly the situation which leads to the lowest $C_{a_{j}}^{2}$ for a given $\lambda_{j}$ ). If we substitute these values for $C_{a_{j}}^{2}$ in expressions (17)-(18) and if we take the limit for $\lambda_{j} \rightarrow 0$ (for given mean service times, the mean delay is minimal when the arrival rates approach zero), the first two terms of these equations then both equal $\mu_{j} / 2(j=1,2)$ respectively. This indeed leads to a minimal mean class $-j$ packet delay of $\mu_{j}$.

In Tables 1 and 2, the dependence of the mean delay of both priority classes on the different parameters of class 1 and class 2 packet arrival and service processes is summarized qualitatively. The parameter in question is varied while the others are kept constant. Note that the parameters $\rho_{j}, \mu_{j}$ and $\lambda_{j}$ are related by $\rho_{j}=\lambda_{j} \mu_{j}(j=1,2)$.

The parameter $\rho_{a_{1} a_{2}}$ in expressions (17)-(18) represents the correlation between the number of per-slot arrivals of both classes. Positive correlation leads 


\begin{tabular}{||c|l|l||}
\hline class-2 parameters & $\mathrm{E}\left[d_{1}\right]$ & $\mathrm{E}\left[d_{2}\right]$ \\
\hline$\rho_{2}\left(\mu_{2}\right.$ constant $)$ & $\sim \rho_{2}$ & $\sim 1 /\left(1-\rho_{1}-\rho_{2}\right)$ \\
\hline$\rho_{2}\left(\lambda_{2}\right.$ constant $)$ & $\sim \rho_{2}+A_{2} \rho_{2}^{2}$ & $\sim \rho_{2}+B_{2} /\left(1-\rho_{1}-\rho_{2}\right)$ \\
\hline$\mu_{2}\left(\rho_{2}\right.$ constant $)$ & $\sim \mu_{2}$ & $\sim \mu_{2}$ \\
\hline$C_{a_{2}}$ & independent & $\sim C_{a_{2}}^{2}+C_{2} C_{a_{2}}$ \\
\hline$C_{s_{2}}$ & $\sim C_{s_{2}}^{2}$ & $\sim C_{s_{2}}^{2}$ \\
\hline & &
\end{tabular}

Table 2

Dependence of the mean delay of both priority classes on the system parameters of the low-priority class $\left(A_{2}, . ., C_{2}\right.$ are constants)

to a larger delay of class-2 packets. The opposite holds for a negative correlation between the number of per-slot arrivals of both classes. The delay of high-priority packets is independent of $\rho_{a_{1} a_{2}}$. This is evident, because higher $\rho_{a_{1} a_{2}}$ causes a higher probability that both high- and low-priority packets arrive during the same slot. The high-priority packets have to be served before these low-priority packets, which results in a larger low-priority mean delay, while the mean delay of the high-priority packets is not influenced by the low-priority packets that arrive in the same slot, and thus is not influenced by $\rho_{a_{1} a_{2}}$.

Finally, defining $\alpha$ as the fraction of high-priority load in the overall load, and substituting $\rho_{1}$ and $\rho_{2}$ by $\alpha \rho_{T}$ and $(1-\alpha) \rho_{T}$ respectively in (17) and (18), we can analyze the influence of $\alpha$ on the mean delay of both classes. The behavior of the mean delay of both priority classes as a function of $\alpha$ is shown in Figure 2, for $\rho_{T}=0.5, \mu_{1}=\mu_{2}=2, C_{a_{1}}=C_{a_{2}}=0.5, \rho_{a_{1} a_{2}}=-0.5, C_{s_{2}}=0$ and $C_{s_{1}}=0, \sqrt{0.125}, 0.4,0.6$ and 0.9 respectively. This figure illustrates the following behavior. For notational purposes, define $E \triangleq C_{a_{1}}^{2}+\mu_{1} C_{s_{1}}^{2}, F \triangleq$ $\left(1-\rho_{T}\right)\left(\mu_{2} C_{s_{2}}^{2}+\left(\mu_{2}-1\right)\right)$ and $G \triangleq\left(C_{a_{1}}-C_{a_{2}}\right)^{2}+2 C_{a_{1}} C_{a_{2}}\left(1-\rho_{a_{1} a_{2}}\right)$. One can show that if $E=F$, the mean delay of class-1 is independent of $\alpha$. If $E>F$, the mean delay of class-1 increases when $\alpha$ becomes larger. If $E<F$, the mean delay of class-1 decreases when the fraction of the high-priority load becomes larger. The influence of $\alpha$ on the mean delay of the low-priority class is a bit more involved. $\alpha$ has no influence on the mean low-priority delay when $E=F+G$. When $E>F+G$, the mean low-priority delay grows with $\alpha$. If $E<F+\left(1-\rho_{T}\right)^{2} G, \mathrm{E}\left[d_{2}\right]$ becomes smaller when $\alpha$ is larger. Finally, when $F+\left(1-\rho_{T}\right)^{2} G<E<F+G, E\left[d_{2}\right]$ reaches a minimum for $\alpha_{\text {min }}=(1-\sqrt{(E-F) / G}) / \rho_{T}$. When $\alpha<\alpha_{\text {min }}, E\left[d_{2}\right]$ becomes smaller when $\alpha$ grows. The opposite occurs for $\alpha>\alpha_{\text {min }}$. 


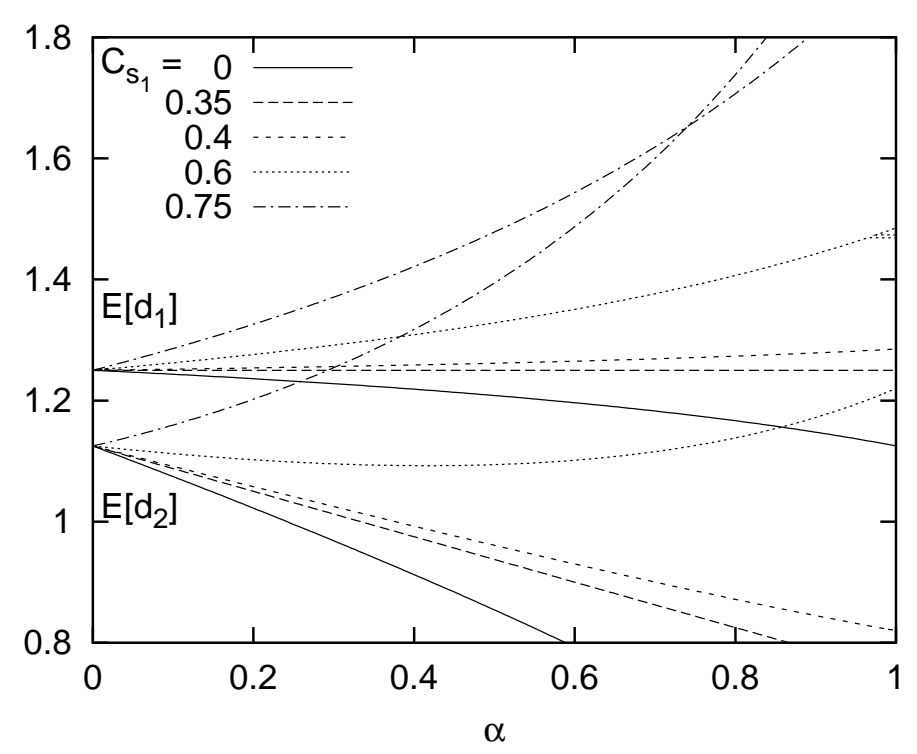

Fig. 2. Mean packet delay versus $\alpha$ for several values of the other parameters

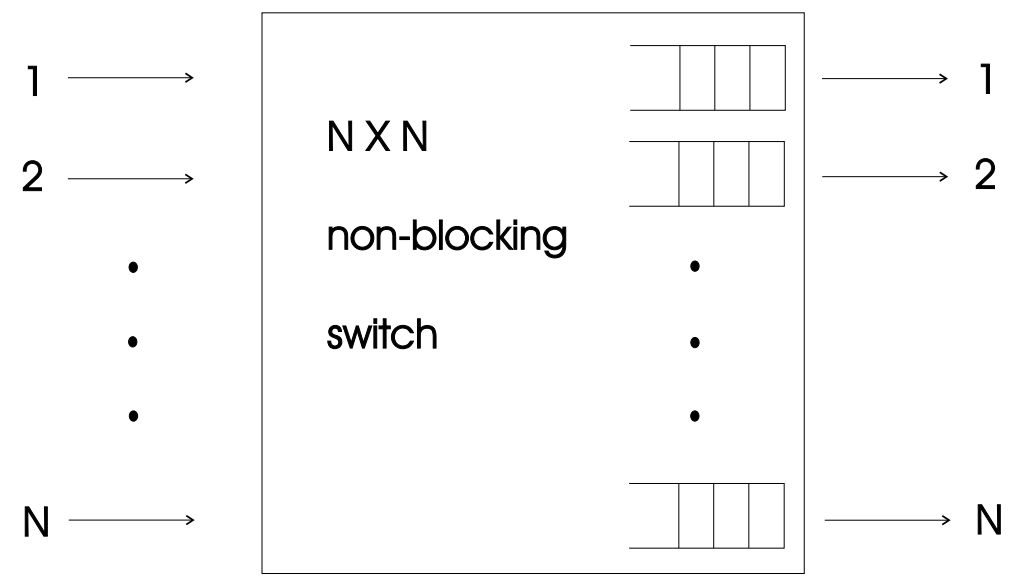

Fig. 3. An $N \mathrm{x} N$ router with output queues

\section{Application}

In this section, we apply our results to an $N \mathrm{x} N$ router with output queues (see Figure 3). We assume two types of traffic. Traffic of class- 1 is delay-sensitive and traffic of class-2 is assumed to be delay-insensitive. We investigate the effect of a non-preemptive priority scheduling discipline, as presented in the former of this paper.

The packet arrivals on each inlet are assumed to be i.i.d., and generated by a Bernoulli process with arrival rate $\lambda_{T}$. An arriving cell is assumed to be of class $j$ with probability $\lambda_{j} / \lambda_{T}(j=1,2)\left(\lambda_{1}+\lambda_{2}=\lambda_{T}\right)$. The incoming cells are then routed to the output queue corresponding to their destination, in an independent and uniform way. Therefore, the output queues behave identically 
and we can concentrate on the analysis of 1 output queue. In view of the previous, the arrivals of both types of packets to an output queue are generated according to a twodimensional binomial process. It is fully characterized by the following joint pgf

$$
A\left(z_{1}, z_{2}\right)=\left(1-\frac{\lambda_{1}}{N}\left(1-z_{1}\right)-\frac{\lambda_{2}}{N}\left(1-z_{2}\right)\right)^{N} .
$$

Obviously, the number of class- 1 and class- 2 arrivals at an output queue during a slot are correlated. This is simply demonstrated by the following observation: when $m$ class- 1 packets arrive at the tagged queue during a slot $(0 \leq m \leq N)$, the maximum number of class-2 arrivals during the same slot is limited by $N-m$. We note that for $N$ going to infinity, the above expression becomes a product of two generating functions, each describing a Poisson process with mean $\lambda_{1}$ and $\lambda_{2}$ respectively, and as a result, the arrival process becomes uncorrelated for both classes. In most of the remainder of this section, we assume that $N=16$.

In the first few figures, we will assume deterministic service times for both classes equal to $\mu_{1}$ and $\mu_{2}$ respectively. In Figures 4 and 5 , the mean value and variance of the packet delay of class- 1 and class- 2 packets is shown as a function of the total load $\rho_{T}$, when $\mu_{1}=\mu_{2}=2$. The parameter $\alpha$, which as before denotes the fraction of the load of class-1 packets of the total load is $0.25,0.5$ and 0.75 respectively. In order to compare with FIFO scheduling, we have also shown the mean value and variance of the packet delay in that case. Since, in this example, the service times of the class- 1 and class- 2 packets are equal, the packet delay is then of course the same for class- 1 and class- 2 packets in case of FIFO scheduling, and can thus be calculated as if there is only one class of packets arriving according to an arrival process with pgf $A(z, z)$. This situation has already been analyzed, e.g., in [2]. One can observe the influence of priority scheduling: mean value and variance of the delay of high-priority packets reduces significantly. The price to pay is of course a larger mean value and variance of the delay for class- 2 packets. If this kind of traffic is not delay-sensitive, as assumed, this is not a too big a problem. Also, for this parameter set, the smaller the fraction of high-priority packets in the overall traffic mix, the lower the mean value and variance of the packet delay of both classes will be. This is not always true however, as discussed in subsection 6.3 and as can be deduced from Figure 6, which shows the mean delay of class- 1 and class- 2 packets as a function of $\rho_{T}$, when $\mu_{1}=2$ and $\mu_{2}=20$. $\alpha$ is again $0.25,0.5$ and 0.75 respectively. In this case, if the load is smaller than $\approx 0.9$, the smaller the fraction of high-priority packets in the overall traffic mix, the higher the mean packet delay of both classes will be. For loads higher than 0.9, the opposite holds. This can be explained as follows. For low and moderate values of the load, due to the long service times of class-2 packets, the delay of class- 1 packets will be determined by the probability of having to wait for a class-2 residual service time upon arrival and is therefore highest 


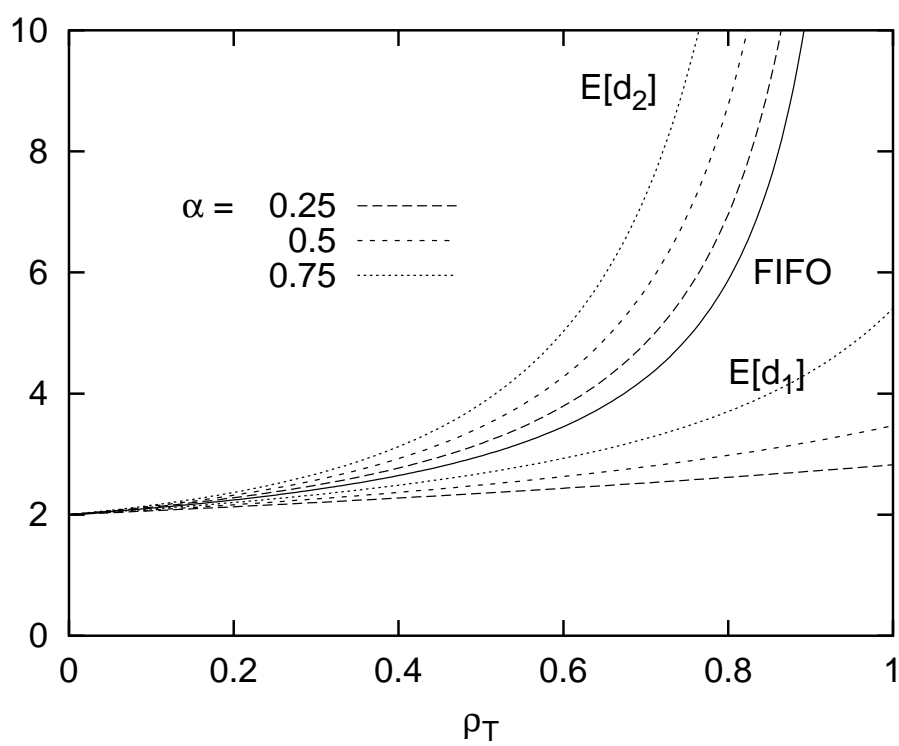

Fig. 4. Mean packet delay versus the total load

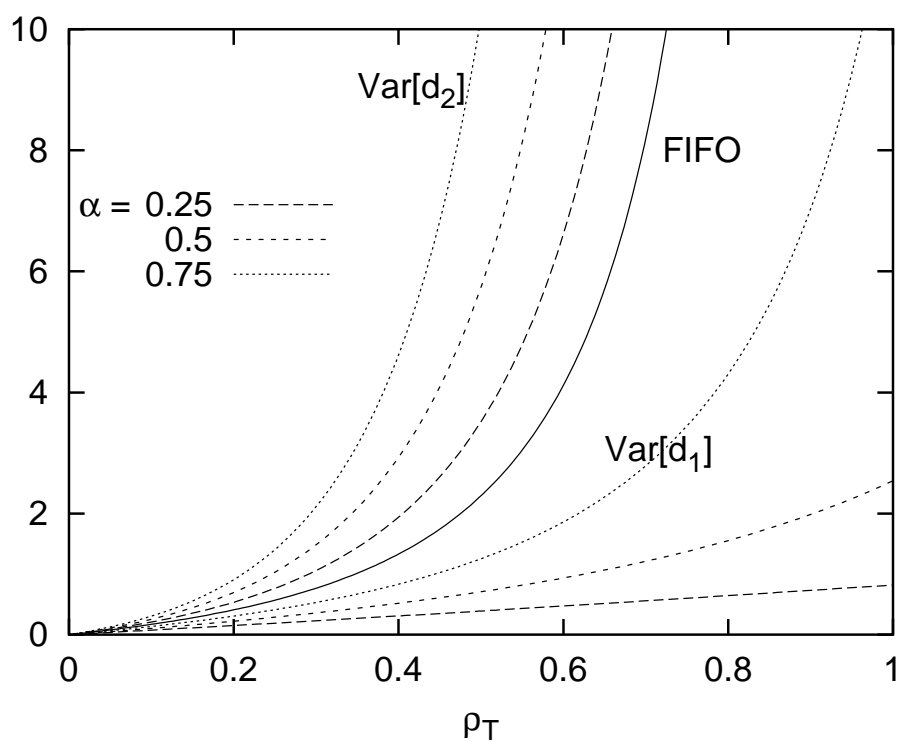

Fig. 5. Variance of the packet delay versus the total load

when the share of the class- 2 packets in the overall traffic mix is highest. The same holds for the delay of the class- 2 packets since there are too few class1 packet arrivals to have a severe impact on their delay. As the total load further increases however, the transmission of high priority packets becomes more frequent and starts to take its toll.

Figure 7 shows the mean packet delay of high and low-priority packets as a function of the service time of high-priority packets, when $\rho_{T}=0.75, \mu_{2}=2$ and $\alpha$ is, as before, $0.25,0.5$ and 0.75 . We see that the mean packet delay of both classes is proportional with $\mu_{1}$ and that the impact of $\mu_{1}$ on the delay of 


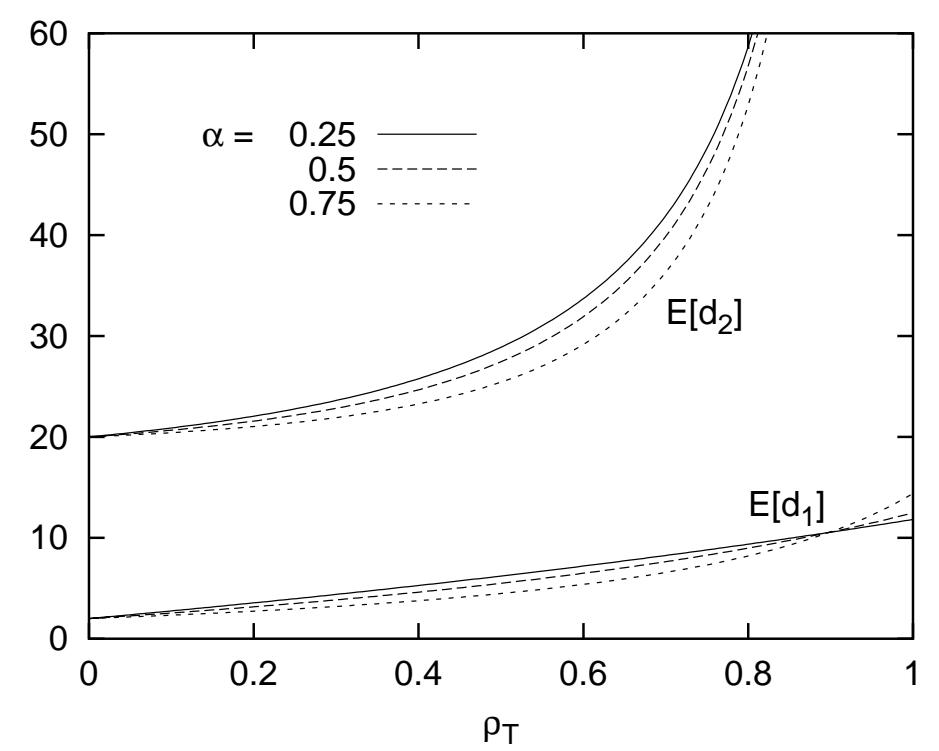

Fig. 6. Mean packet delay versus the total load

both priority classes is significant. Figure 8 shows the mean packet delay of high and low-priority packets as a function of the service time of low-priority packets, when $\rho_{T}=0.75, \mu_{1}=2$ and $\alpha$ is, as before, $0.25,0.5$ and 0.75 . Varying the mean service time of low-priority packets has a considerable influence on the delay of low-priority packets themselves, while the influence on the mean packet delay of high-priority packets is small, but not neglegible. Furthermore we observe that for short low-priority packet lenghts, both classes have a smaller delay if the fraction of the load of high-priority packets decreases, while for long service times of the low-priority packets, the opposite holds, as already discussed before (see also Figure 6). In addition, as indicated in Tables 1 and 2 and as can be concluded from Figures 7 and 8, the mean delay of both priority classes is proportional to $\mu_{1}$ and $\mu_{2}$, given that $\rho_{1}$ and $\rho_{2}$ are kept constant.

Figure 9 shows the mean value of the packet delay of high-priority packets as a function of the total load, when $\lambda_{1}=0.25, \mu_{1}=2$ and $\mu_{2}=1,2,4,8,16$. This figure shows the influence of the non-preemptive priority scheduling. When the service time of a class- 2 packet is assumed to be deterministically 1 slot, i.e., $\mu_{2}=1$, the non-preemptive priority scheduling has the same effect as the preemptive priority scheduling, and $\mathrm{E}\left[d_{1}\right]$ is not influenced by the presence of low-priority packets. For $\mu_{2}>1$, the larger the value of $\rho_{T}$ (and hence $\rho_{2}$ ), the larger the probability that a newly arriving high-priority packet will have to wait for a low-priority packet service completion, and obviously the effect becomes worse as $\mu_{2}$ increases. Furthermore, for a given value of the low-priority packet length, the mean high-priority packet delay increases proportional to the total load $\rho_{T}$, as can be deduced from Tables 1 and 2 .

In the next two figures, we assume the service time of class- 1 or class- 2 packets 


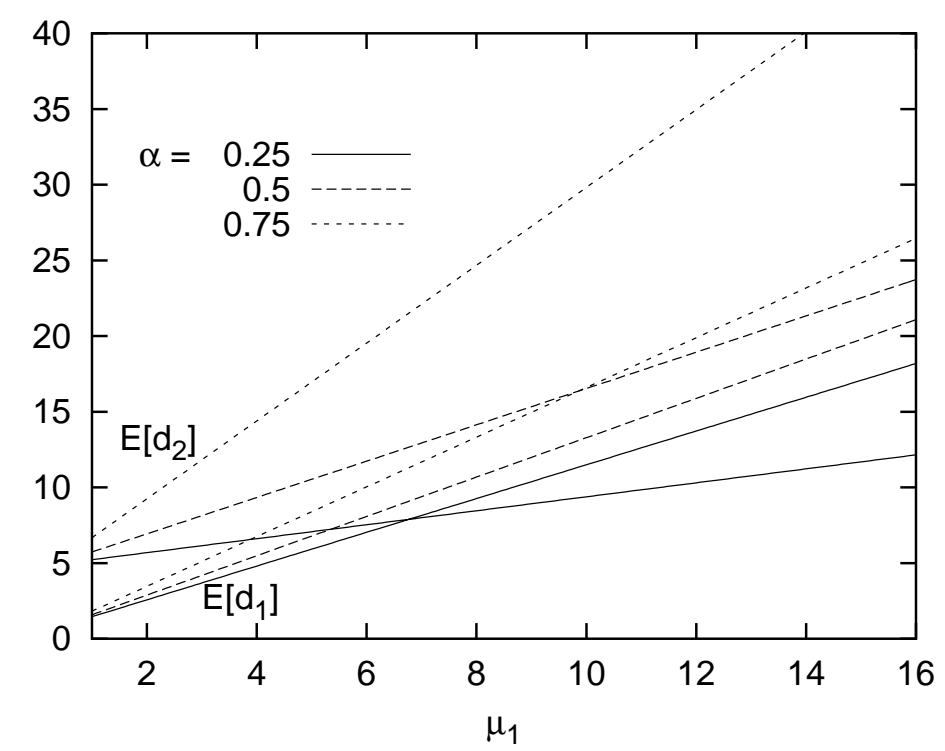

Fig. 7. Mean packet delay versus mean service times of class-1 packets

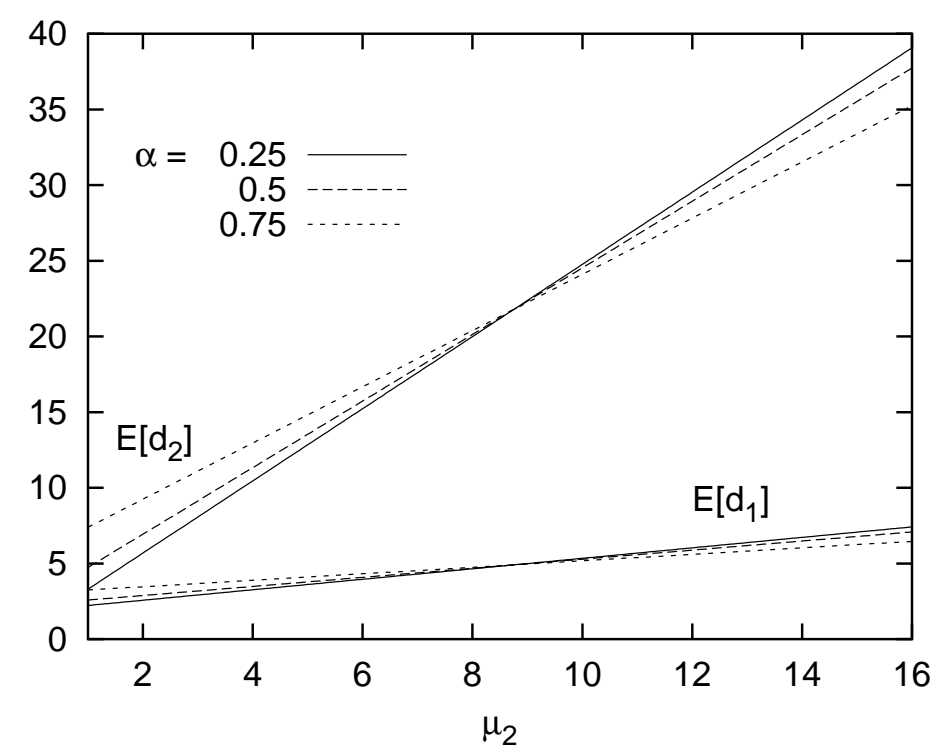

Fig. 8. Mean packet delay versus mean service times of class-2 packets

respectively to be a weighted sum of two geometric distributions (the service times of the packets of the other class remain deterministic), i.e.,

$$
S_{j}(z)=p \frac{z}{\mu_{j, 1}-\left(\mu_{j, 1}-1\right) z}+(1-p) \frac{z}{\mu_{j, 2}-\left(\mu_{j, 2}-1\right) z},
$$

with $j=1,2$. The weight is chosen in such a way that the the mean service time remains constant, i.e., $\mu_{j}=p \mu_{j, 1}+(1-p) \mu_{j, 2}=5$. In the remainder, we will set $\mu_{j, 1}=2$, while $\mu_{j, 2}$ can take any value between 5 and $\infty$. The coefficient of variation $C_{s_{j}}$ then varies accordingly from $\sqrt{0.8}$ to $\infty$. In Figure 10 , we have plotted the mean delay of both priority classes as a function of the 


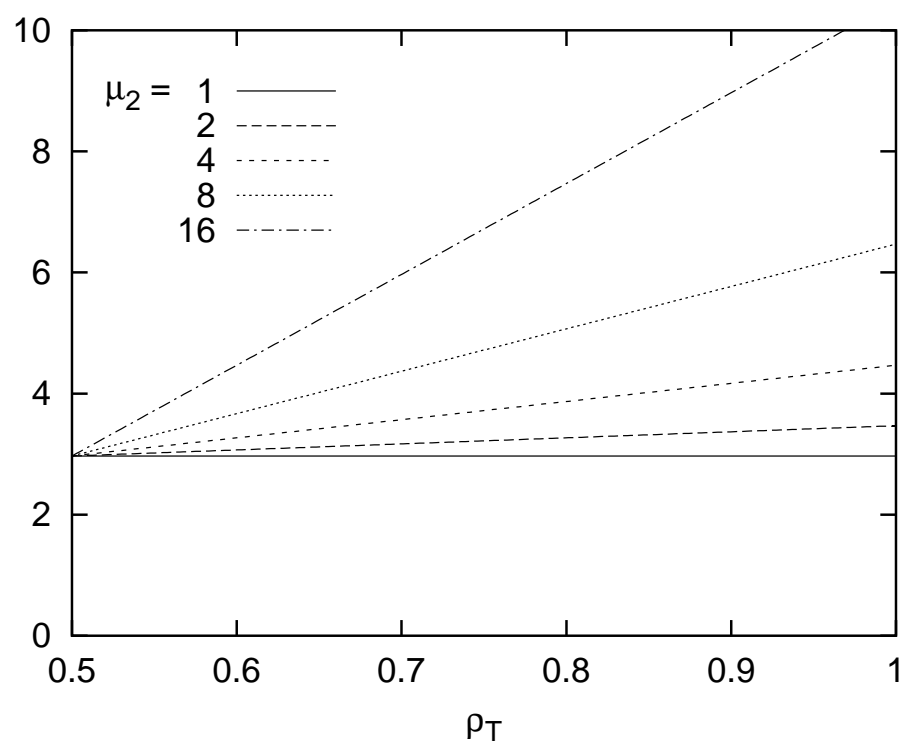

Fig. 9. Mean packet delay of class-1 packets versus the total load

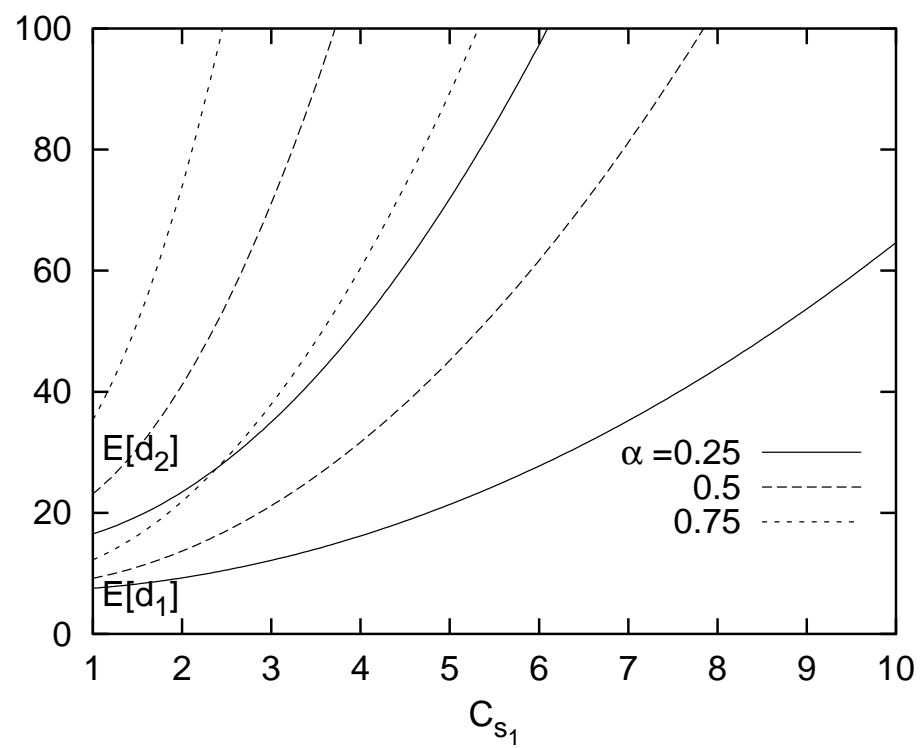

Fig. 10. Mean packet delay of both classes versus the coefficient of variation of the class-1 service times

coefficient of variation of the service times of class- 1 packets, when $\rho_{T}=0.75$, $\mu_{1}=\mu_{2}=5$ and $\alpha=0.25,0.5$ and 0.75 respectively. Figure 11 shows the mean delay of both priority classes as a function of the coefficient of variation of the service times of class- 2 packets, when $\rho_{T}=0.75, \mu_{1}=\mu_{2}=2$ and $\alpha=0.25,0.5$ and 0.75 respectively. These figures illustrate that even though the mean lengths of high- and low-priority packets are kept constant, the variability of the latter quantities still has a huge impact on both the highand low-priority mean packet delay.

In Figure 12, the mean delay of both classes is shown as a function of the 


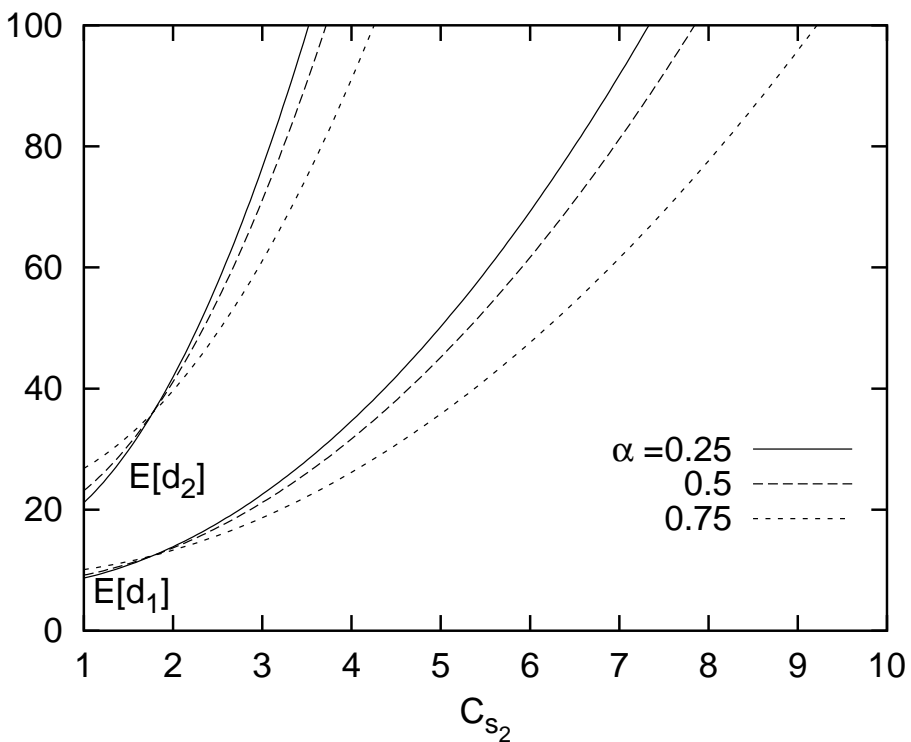

Fig. 11. Mean packet delay of both classes versus the coefficient of variation of the class-2 service times

number of in- and outlets $N$, for deterministic service times of 2 slots. We have also plotted the mean delay of the low-priority packets when the correlation between the number of arrivals of both classes is neglected, i.e., $\rho_{a_{1} a_{2}}$ is zero (in which case the joint pgf of the number of packet arrivals of both classes is set equal to the product of their respective marginal pgf's). The figure shows that the mean delay of both classes grows with the number of inlets for approximately $N<16$ and stays constant for higher $N$. It also shows that if the correlation between the number of arrivals of both classes was neglected there would be an overestimation of the mean low-priority delay for $N<16$. For higher number of inlets, this correlation factor is neglegible. Since $E\left[d_{1}\right]$ does not depend on $\rho_{a_{1} a_{2}}$, the curves for the mean high-priority packet delay are the same with or without the correlation.

To conclude this section, we analyze the following case-study. Assume we have two priority classes arriving to a buffer with joint pgf given by expression (20). The service times of all packets are equal to 2 . The high-priority class has the following constraint for its mean delay: $\mathrm{E}\left[d_{1}\right]<T_{1}$, where $T_{1}$ is a parameter dependent on the application. The delay of low priority packets has a similar constraint: $\mathrm{E}\left[d_{2}\right]<T_{2}$. The latter constraint is however not as severe as the first one (i.e. $T_{1}<T_{2}$ ), because the low-priority traffic is in general not as delay-sensitive as the high-priority traffic. The question we wish to answer is the following: what is the maximal load $\rho_{T, \max }$, as a function of $\alpha$, that still fulfils the two constraints? In Figure 13, we show the maximal load as a funtion of $\alpha$ when $T_{2}=20$ and various values of $T_{1}$. For $T_{1}>5$, we observe that $T_{2}$ imposes the decisive constraint for virtually all values of $\alpha$. For lower $T_{1}$ however, the constraint for the mean delay of the high-priority traffic becomes decisive for high $\alpha$, i.e. when more class-1 packets arrive. In Figure 14, the 


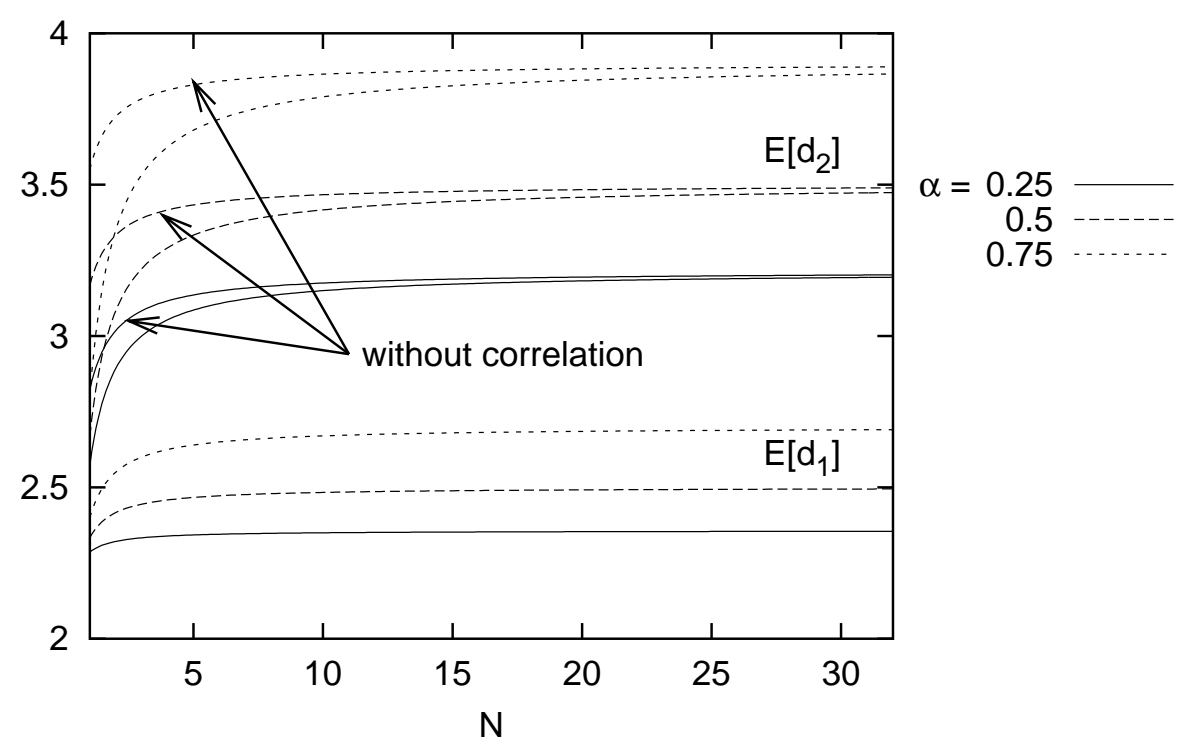

Fig. 12. Mean packet delay of both classes versus the number of inlets

maximum tolerable load as a function of $\alpha$ is shown, when $T_{1}=3$ and several values of $T_{2}$. For low $T_{2}$ and low $\alpha$ the constraint for the mean low-priority delay is the most stringent one. For higher $T_{2}$ and/or higher $\alpha$, the constraint for the mean high-priority delay becomes dominant. The behavior depicted in these two figures can be explained as follows. For $\alpha=0$, the traffic mix consists of low-priority packets only, and $\rho_{T, \max }$ is relatively high, depending on the value of $T_{2}$. As $\alpha$ increases, $\rho_{T, \max }$ gradually decreases (but is still determined by $T_{2}$ ) since the growing fraction of high-priority packets causes the mean low-priority packet delay to rise. Then, as $\alpha$ further increases, a transition point is reached, which is defined as the value of $\alpha$ and $\rho_{T}$ for which $\mathrm{E}\left[d_{1}\right]=T_{1}$ and $\mathrm{E}\left[d_{2}\right]=T_{2}$. Beyond this transition point, the bounding set by $T_{1}$ becomes predominant, and $\rho_{T, \max }$ further decreases due to the ever increasing presence of high-priority packets in the traffic mix. These figures show that the maximum allowable load can strongly depend on the delay boundaries $T_{1}$ and $T_{2}$ set on the high- and low-priority mean packet delays, and the traffic mix $\alpha$.

Similar plots as the ones shown in this section can also be made for higher order moments, such as the variance. However, the curves that are thus obtained are quite similar, and the conclusions that can be drawn remain the same.

\section{Conclusions}

In this paper, we analyzed the high- and low-priority packet delay in a queueing system with non-preemptive HOL priority scheduling. A generating-functions- 


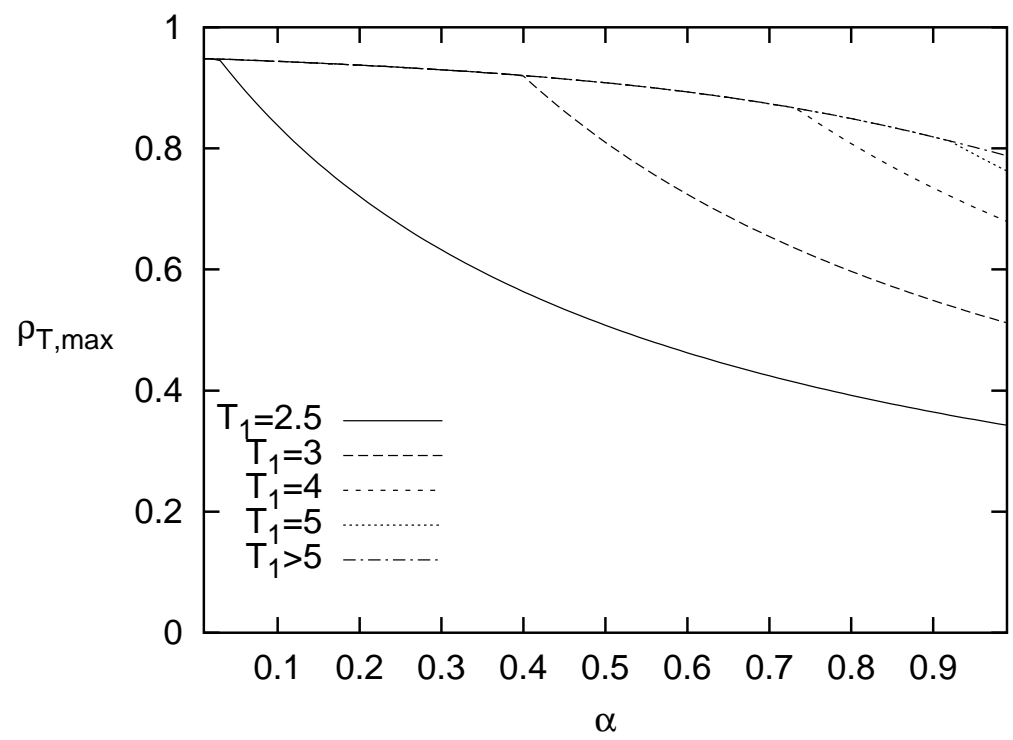

Fig. 13. Maximum load versus the fraction of class-1 arrivals for several values of $T_{1}$

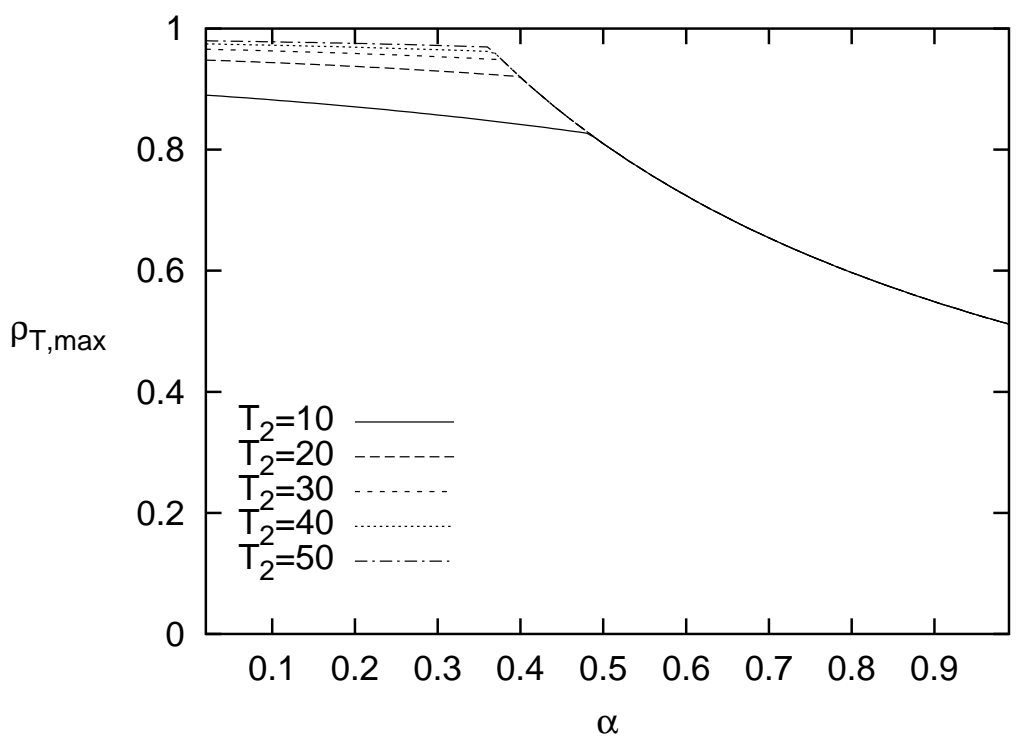

Fig. 14. Maximum load versus the fraction of class-1 arrivals for several values of $T_{2}$

approach was adopted, which led to closed-form expressions for some of the relevant performance measures, such as the mean of packet delay of both classes, that are easy to evaluate. The model included possible correlation between the number of arrivals of the two classes during a slot, and general service times for packets of both classes. The results could be used to analyze performance of buffers in a packet-based networking context. As an application, we therefore studied the performance of an output queueing switch/router by means of extensive numerical examples. 


\section{References}

[1] J.J. Bae and T. Suda, Survey of traffic control schemes and protocols in ATM networks, Proceedings of the IEEE 79(2), pp. 170-189, 1991.

[2] H. Bruneel and B.G. Kim, Discrete-time models for communication systems including ATM, Kluwer Academic Publishers, Boston, 1993.

[3] A. Khamisy and M. Sidi, Discrete-time priority queueing systems with two-state Markov modulated arrival processes, Stochastic Models 8(2), pp. 337-357, 1992.

[4] K. Laevens and H. Bruneel, Discrete-time multiserver queues with priorities, Performance Evaluation 33(4), pp. 249-275, 1998.

[5] K. Liu, D.W. Petr, V.S. Frost, H. Zhu, C. Braun and W.L. Edwards, Design and analysis of a bandwidth management framework for ATM-based broadband ISDN, IEEE Communications Magazine, pp. 138-145, 1997.

[6] L. Kleinrock, Queueing systems volume II: Computer applications, John Wiley \& Sons, 1976.

[7] R.G. Miller, Priority queues, Annals of Mathematical Statistics, pp. 86-103, 1960.

[8] I. Rubin and Z. Tsai, Message delay analysis of multiclass priority TDMA, FDMA, and discrete-time queueing systems, IEEE Transactions on Information Theory 35(3), pp. 637-647, 1989.

[9] D.A. Stanford, Interdeparture-time distributions in the non-preemptive priority $\sum M_{i} / G_{i} / 1$ queue, Performance Evaluation 12, pp. 43-60, 1991.

[10] A. Sugahara, T. Takine, Y. Takahashi and T. Hasegawa, Analysis of a nonpreemptive priority queue with SPP arrivals of high class, Performance Evaluation 21, pp. 215-238, 1995.

[11] H. Takagi, Queueing analysis: A foundation of Performance Evaluation Volume 1: Vacation and priority systems, Elsevier North-Holland, 1991.

[12] T. Takine, Y. Matsumoto, T. Suda and T. Hasegawa, Mean waiting times in nonpreemptive priority queues with Markovian arrival and i.i.d. service processes, Performance Evaluation 20, pp. 131-149, 1994.

[13] T. Takine, B. Sengupta and T. Hasegawa, An analysis of a discrete-time queue for broadband ISDN with priorities among traffic classes, IEEE Transactions on Communications 42 (2-4), pp. 1837-1845, 1994.

[14] T. Takine, A nonpreemptive priority $M A P / G / 1$ queue with two classes of customers, Journal of Operations Research Society of Japan 39(2), pp. 266290, 1996.

[15] P. Van Mieghem, B. Steyaert and G.H. Petit, Performance of cell loss priority management schemes in a single server queue, International Journal of Communication Systems 10, pp. 161-180, 1997. 
[16] X. Xiao and L.M. Ni, Internet QoS: a big picture, IEEE Network 13 (2), pp. 8-18, 1999.

[17] J. Walraevens and H. Bruneel, HOL priority in an ATM output queueing switch, Proceedings of the seventh IFIP workshop on performance modelling and evaluation of ATM/IP networks, Antwerp, 1999. 